\title{
Aproximación en torno a la adecuación del esfuerzo terapéutico en pacientes pediátricos con patología neurológica grave y dependientes de ventilación mecánica crónica (I parte)
}

\author{
HUGO A. CERDA B.*
}

Fitness of therapeutic effort in pediatric patients with severe neurological pathology and depending on mechanical ventilation (Part I)

The advancement of technology has led to the emergence of ethical dilemmas in patients who, despite their condition, have remained alive with a poor quality of life. The fitness of therapeutic effort is proposed as a way to avoid disproportionate therapies and provide appropriate measures in patients with chronic or terminal illnesses.

Key words: Limitation/fitness of therapeutic effort; quality of life.

\section{Resumen}

El avance de la tecnología ha motivado la aparición de dilemas éticos en pacientes, que pese a su patología, se han mantenido vivos con una mala calidad de vida. Se plantea la adecuación del esfuerzo terapéutico (AET), como una forma de evitar terapias desproporcionadas y aportar las medidas útiles en pacientes con enfermedades crónicas o terminales.

Palabras clave: Adecuación/limitación del esfuerzo terapéutico, proporcionalidad, calidad de vida.

En las últimas décadas, el avance de la tecnología a niveles insospechados junto con el aumento de la secularización de la sociedad, han permitido la sensación de que el hombre (médico, científico) puede controlar y modificar la vida humana. De esta manera, hemos sido testigos del control y reversión en la evolución de patologías cuya mortalidad era indiscutible y aceptada como normal hasta hace unos años. La muerte se ha convertido en un fin por combatir y quizás por extinguir (anhelada por algunos). Esta falacia, a su vez, ha trascendido a la sociedad, donde la medicina, lamentablemente ha transformado a algunos pacientes en clientes, quienes no se conforman con un mal pronóstico y atribuyen los malos resultados, a la mala práctica de los médicos. Es decir, la sensación de autosuficiencia y dominio de la naturaleza se ha convertido en una herramienta de mayor inseguridad ante el destino del hombre. La muerte ya no es el fin natural del hombre, sino que es vista en una connotación más negativa, con desesperanza, ya que se duda que exista alguna forma de trascendencia de tipo religioso, lo que obliga a aferrarse a la vida, tenida como lo único seguro.

En el caso de los niños, la situación descrita es más compleja. El cariño natural que brindan sus padres, genera una carga emocional que torna más difícil la aceptación de malas noticias. Podemos ahondar, más aún, en los pacientes con patologías neurológicas que involucran un compromiso cognitivo y sensorial severo. Un porcentaje de ellos logran recuperarse, luego de una terapia de rehabilitación, con calidad de vida adecuada. Sin embargo, en muchos otros casos es posible dudar de la real utilidad de indicar

* Pediatra Broncopulmonar, Diplomado en Bioética. Miembro de la Comisión de Ética, Sociedad Chilena de Enfermedades Respiratorias.

Nota de la redacción: En el próximo número de la revista, se publicará la segunda parte de este artículo. 
intervenciones cuyo resultado, en el mejor de los casos, consiste en mantener un niño con estado vegetativo persistente ${ }^{1-3}$.

De esta manera, ha surgido un número cada vez mayor de casos clínicos de mayor complejidad, donde las soluciones se presentan cada vez más difíciles, y en las cuales el "camino fácil" parece ser el deseo de mantener la vida, a toda costa. El médico, pues, opta por mantener la vida de un paciente, más allá de lo propio de la historia natural de la enfermedad, para satisfacer los deseos de una familia que exige ese tipo de resultados.

Por su parte, la bioética nos enseña que el principio de la beneficencia, en el sentido de buscar siempre el "mayor beneficio" para el paciente, no siempre es sinónimo de entorpecer los procesos naturales, para evitar una muerte que a todas luces igualmente se producirá ${ }^{4}$.

La "obstinación terapéutica", que ha venido a reemplazar al "término "encarnizamiento terapéutico" emerge como producto de múltiples factores que se derivan del sistema de valores, creencias y hábitos que integran la cultura de este tiempo en la que la muerte no es aceptada. La determinación del diagnóstico de terminalidad en una Unidad de Cuidados Intensivos Pediátricos (UCIP) es en muchas ocasiones una condición de extrema dificultad, ya que implica establecer con la mayor seguridad posible que un paciente es irrecuperable. Más difícil es aún al considerar que los niños tienen una gran capacidad de recuperación $\mathrm{y}$, muchas veces, la evolución de las enfermedades infantiles es de sorprendente recuperación, tanto en el pronóstico vital como en las potenciales secuelas ${ }^{1,2}$.

Por tanto, se hace cada vez más necesario intentar racionalizar la toma de decisiones para avanzar en la aplicación de criterios aceptables por parte de todos los actores del proceso médico. La bioética brinda distintos métodos o principios para hacer análisis, como la deontología (obligaciones que impone la actividad que realiza), el consecuencialismo (las conductas se miden en base a sus resultados) y por último, el principialismo, actualmente el más invocado porque incluye la beneficencia, no maleficencia, autonomía y justicia ${ }^{6}$.

En la práctica, es posible que deba utilizarse más de un método. Sin embargo, este debe superar el enfoque paternalista de la medicina y consensuar la participación de la familia y del paciente, especialmente cuando tenga posibilidad de tomar conciencia de su enfermedad o de sus consecuencias, en la toma final de decisiones ${ }^{5,7-9}$.

El análisis "caso a caso" hace mención de la inmensa dificultad de sistematizar consensos para aclarar lo que se debe hacer en cada patología y cada paciente. Al no haber acuerdos previos, las discusiones terminan teniendo carácter utilitario en el sentido que se privilegian pacientes con aparente mejor pronóstico al momento de otorgar un cupo en una UCIP, habitualmente superadas en su capacidad. De esta manera al niño portador de secuelas neurológicas, tiende a restringírsele su ingreso a UCIP, así como la posibilidad de recibir tratamientos que puedan considerarse desproporcionados en un momento dado de su evolución.

Toda vida humana es merecedora de respeto y cuidados, pero estos no deben utilizarse a toda costa cuando lo mejor que puede ofrecerse al paciente es un estado vegetativo persistente. Por otra parte, cuando un paciente pese a su daño neurológico es capaz de interaccionar de alguna forma (expresar agrado/desagrado o alguna forma de expresión verbal o motora de tipo voluntaria) con su familia o con otras personas que lo cuidan, facilita aún más la posibilidad para tratarlo con recursos que al menos permitan mantener ese importante vínculo.

El concepto de limitación del esfuerzo terapéutico hace referencia a la decisión clínica de no iniciar o de retirar las medidas de soporte vital cuando se percibe una desproporción entre estas $\mathrm{y}$ los fines a conseguir ${ }^{7-10}$. El objetivo del médico se transforma, entonces, en facilitar la evolución de la enfermedad hacia la muerte natural, sin prolongar la fase agónica y evitando de este modo un tratamiento inútil.

Sin embargo, el uso del concepto de limitación del esfuerzo terapéutico corre el riesgo de desvirtuarse hacia conductas meramente de tipo restrictivo, que más bien facilitan un alejamiento de los cuidados necesarios para el paciente. Es por ello que actualmente se prefiere hablar de "adecuación del esfuerzo terapéutico", como una forma de darle una connotación más dinámica y más activa por parte del médico y del personal hacia todos aquellos cuidados, asistencias y medicamentos que necesite el paciente en cada etapa de su enfermedad.

Se trata entonces de concebir que la conducta éticamente correcta es la que asegura la aplicación de todas las medidas terapéuticas útiles disponibles, sin prolongar situaciones de sufrimiento para el paciente y su familia. Por ende, el objetivo asistencial es restaurar el estado de salud del paciente y cuando esto no es posible, optimizar la calidad de vida en la fase terminal de la enfermedad.

Para abordar adecuadamente el concepto de 
calidad de vida de un paciente se consideran dos vertientes. Una basada en criterios objetivos o estándar de mala calidad de vida, como son la esperanza de vida, el grado futuro de dependencia de los cuidados médicos, la posibilidad de llevar una vida autónoma, la presencia de una mínima capacidad para establecer relaciones de comunicación con el entorno y su nivel de sufrimiento. Y otra subjetiva, basada en los valores del individuo y su familia ${ }^{3,9}$.

Los pasos que se recomiendan para iniciar una limitación del esfuerzo terapéutico en este tipo de pacientes son: hablar con la familia y el paciente sobre la enfermedad, sus síntomas, su pronóstico y las posibilidades reales de reversibilidad del proceso, acompañar a la familia y contestar en todo momento las dudas que surjan. En segundo lugar, una vez aceptada la nueva realidad por parte de la familia, se continúa con el tratamiento sin añadir nuevas acciones y, se fijan plazos de evaluación para decidir cuando procede su retirada. Se podrá plantear la supresión de algún tratamiento, así como la retirada gradual de ventilación asistida si procede. Se consideran actos fútiles el ingreso en la UCIP, la reanimación cardio-pulmonar (RCP) avanzada, la administración de antibióticos ante complicaciones infecciosas o de quimioterapia en situación terminal. En cuanto a la orden de no reanimar, siempre consensuada con la familia, debe constar de forma visible en la historia clínica para el conocimiento del resto de profesionales implicados en el cuidado del paciente.

En los escenarios que estamos analizando, la fundamentación ética basada en los cuatro principios fundamentales bioéticos se puede entender de la siguiente manera:

a) No maleficencia: se evitan actuaciones médicas inútiles o lesivas, como realizar maniobras de RCP en pacientes terminales, prolongando una situación de agonía innecesariamente.

b) Beneficencia: tratamiento sintomático paliativo. Manejo del dolor y procurar el mejor confort disponible.

c) Respeto a la autonomía: en pediatría se basa en la subrogación en la toma de decisiones a sus padres o representantes legales.

d) Justicia: la escasez de recursos nunca debe fundamentar las decisiones, pero el uso proporcional y prudente de los recursos disponibles sí es una responsabilidad que debe considerar el médico.

Hemos podido observar que la sociedad ha variado el criterio desde pedir la máxima eficacia, hacia solicitar la máxima ayuda en base al respeto de la voluntad del paciente y el conocimiento de sus necesidades $4,5,7,8,9,13,15,18$.

En este contexto, y más allá de los pacientes pediátricos, la aplicación de limitación del esfuerzo terapéutico se ha convertido en una de las decisiones más complejas a las que hoy en día se enfrenta el profesional en la atención a las personas enfermas. Por ello, existen numerosos esfuerzos por avanzar en la sistematización de las pato-

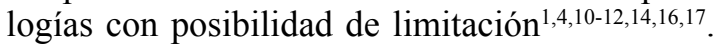
Sin embargo, las múltiples realidades jurídicas no hacen aplicables del todo tales recomendaciones en nuestro país. En el año 1999, en Argentina, el Comité Nacional de terapia intensiva pediátrica ${ }^{11}$, redactó un "consenso sobre recomendaciones acerca de la limitación del soporte vital en terapia intensiva". En este documento intenta tipificar patologías específicas que limitarían su ingreso a UCI y las agrupa de la siguiente manera:

Pacientes terminales: con evolución progresiva e irreversible, sin medidas terapéuticas efectivas y con inminencia de muerte.

Pacientes con pobre calidad de vida, separándolos a su vez en dos categorías:

a) Coma o estado vegetativo persistente, sin interacción con el medio.

b) Pacientes con incapacidad grave, que dependen de otros para realizar todas las actividades cotidianas. Incluye el daño neurológico severo (parálisis cerebral; secuela de traumatismos, infecciones, etc.) o la incapacidad respiratoria progresiva (enfermedades neuromusculares, enfermedades osteoarticulares deformantes). Es en este último punto donde acepta la realidad de discutir "caso a caso", cuando los pacientes no presenten una condición "crítica".

En Chile, no existen consensos amplios en este sentido. Cada hospital maneja sus propios criterios de admisibilidad, los que no siempre son públicos (por ejemplo página web institucional). En este sentido, un paciente que no ingresa a una UCIP de un hospital puede ingresar a la de otro, con las mismas patologías; $y$, es más, puede darse el caso que el ingreso a la UCIP de un hospital quede en manos del criterio del médico que esté de turno en ese momento.

Se trata entonces de insistir en los esfuerzos mancomunados de médicos clínicos, bioeticistas, y los propios familiares (y pacientes, cuando sea posible) para mantener un diálogo fluido y permanente, considerando incluso la posibilidad de cambiar las decisiones tomadas si la evolución del paciente así lo exige.

Nos parece que los comités de ética asistenciales, debieran tener un papel más activo en al- 
gunos de estos casos. Sería ideal que optimizaran su accesibilidad, pudiendo sesionar, si el caso lo requiere, en cualquier momento del día, al menos con algunos miembros. Ello significa un compromiso profesional de disposición durante las $24 \mathrm{~h}$ del día. Esto lo planteamos con el fin de analizar la toma de decisiones frente a la aparición súbita e inesperada de un dilema ético clínico, en el cual es prioritario tomar una decisión de orden práctico. En estas situaciones no es posible esperar que el comité sesione en horario normal.

Por otra parte, creemos que la persona del médico tratante (otrora de cabecera), con su presencia y actitud constituye un ente fundamental para mantener las confianzas en las distintas decisiones y acuerdos. Un médico que permita la cercanía de los familiares, que maneje la historia clínica, y que además facilite el diálogo activo entre los distintos especialistas y profesionales involucrados en el caso clínico, es a todas luces un muy deseable factor que hará más fácil $\mathrm{y}$ humanizada la aceptación, no sólo de las complejas decisiones que se tomen, sino que también jugará un papel clave a la hora de comprender que se han agotado los esfuerzos terapéuticos en el paciente.

El trabajo con este tipo de pacientes, plantea en clave antropológica, la necesidad, compromiso y disposición de escuchar no sólo una, sino muchas veces a los familiares. Por otra parte, retomar la habilidad y actitud médica de saber consolar, lo que demanda a veces sólo nuestra presencia en el momento indicado, alguna palabra de consuelo, o un abrazo a un paciente o su familia, especialmente cuando se advierte la cercanía de la muerte.

\section{Bibliografía}

1.- NOVOA F. Ética en Neuropediatría. Neurología pediátrica. Editorial Mediterráneo, Santiago, Chile. 2012.

2.- NOVOA F. Dilemas éticos en la limitación terapéutica en edad pediátrica. Rev Chil Pediatr 2008; 79 Supl (1): 51-4.

3.- BARBERO J, ROMEO C, GIJON P, JÚDEZ J. Limitación del esfuerzo terapéutico. Med Clin (Barc) 2001; 117: 586-94.

4.- BÓRQUEZ G, ANGUITA V, BERNIER L. El prematuro en cuidado intensivo neonatal ¿Cuándo es el momento de decir no más? Reflexión bioética en torno a la limitación del esfuerzo terapéutico. Rev Chil Pediatr 2004; 75: 181-187.

5.- LOS REYES M, SÁNCHEZ M. Bioética y pediatría, proyectos de vida plena. Sociedad de Pediatría de Madrid y Castilla-La Mancha. Editorial Ergon Madrid, 2010.
6.- BEAUCHAMP T L, CHILDRESS J F. Principles of Biomedical Ethics. Oxford University Press 1979.

7.- TABOADA P. Limitación del esfuerzo terapéutico y principio ético de proporcionalidad terapéutica, en García J. (director): Enciclopedia de Bioética (2011), Disponible en: http://enciclopediadebioetica.com/ index.php/todas-las-voces/146-limitacion-de-esfuerzoterapeutico

8.- LOLAS F, DE FREITAS J. Bioética. Editorial Mediterráneo. Santiago, Chile. 2013.

9.- BECA J, ASTETE C. Bioética Clínica. Editorial Mediterráneo. Santiago, Chile, 2012.

10.- PEIRÓ A, AUSINA A, TASSO M. Limitación bioética del esfuerzo terapéutico en pediatría. Rev Clin Esp. 2012; 212: 305-7.

11.- ALTHABE M, CARDIGNI G, VASALLO J, CODERMATZ M, ORSI C, SAPORITI A. Consenso sobre recomendaciones acerca de limitación del soporte vital en terapia intensiva. Archivos Argentinos de Pediatría 1999; 97: 411-5.

12.- Grupo de estudios de ética clínica. Sociedad Médica de Santiago. Sobre las acciones médicas proporcionadas y el uso de métodos extraordinarios de tratamiento. Rev Med Chile 2003; 131: 685-92.

13.- ARMIJO P, HURTADO S, MONASTERIO C. Implicancias éticas en el manejo del niño gravemente enfermo atendido en una unidad de paciente crítico pediátrica. Acta Bioethica 2014; 20: 51-9.

14.- TRENCHS V, CAMBRA F, PALOMEQUE A, BALCELLS J, SERIÑÁ C, TEZANOS H. y Grupo de Trabajo en Limitación Terapéutica. Limitación terapéutica en cuidados intensivos. An Esp Pediatr 2002; 57: 511-7.

15.- VIDAL C. ¿Es ético limitar el esfuerzo terapéutico? An Esp Pediatr 2002; 57: 505-7.

16.- CASANUEVA L, RUIZ P, SÁNCHEZ J, RAMOS M, BELDA S, LLORENTE A, et al. Cuidados al final de la vida en la unidad de cuidados intensivos pediátrica. Revisión de la bibliografía. An Pediatr (Barc) 2005; 63: 152-9.

17.- AMERICAN ACADEMY OF PEDIATRICS. Guidelines on Forgoing life-sustaiting medical treatment. Pediatrics 1994; 93: 532-6.

18.- AMERICAN ACADEMY OF PEDIATRICS. Palliative Care for Children Committee on Bioethics and Committee on Hospital Care. Policy Statement: Pediatric Palliative Care and Hospice Care Commitments, Guidelines and Recommendations. Section on Hospice and palliative medicine and Committee on Hospital Care. Pediatrics 2013; 132: 5 966-72.

Correspondencia a:

Dr. Hugo A. Cerda B.

Email: hugocerda2003@yahoo.es 